\title{
New combinations and new and resurrected names in Gaultheria (Ericaceae)
}

\author{
K.A. Kron ${ }^{1}$, P.W. Fritsch ${ }^{2}$, L. Lu ${ }^{3} \&$ W.S. Judd ${ }^{4}$ \\ ${ }^{1}$ Department of Biology, 226 Winston Hall, Box 7325 Reynolda Station, Wake Forest \\ University, Winston-Salem, North Carolina 27109, USA \\ ${ }^{2}$ Botanical Research Institute of Texas, 1700 University Drive, \\ Fort Worth, TX 76107, USA \\ pfritsch@brit.org \\ ${ }^{3}$ School of Pharmaceutical Sciences and Yunnan Key Laboratory of Pharmacology \\ for Natural Products, Kunming Medical University, \\ CN-650500 Kunming, Yunnan, China \\ ${ }^{4}$ Department of Biology, University of Florida, 220 Bartram Hall, \\ P.O. Box 118525, Gainesville, Florida 32611, USA
}

\begin{abstract}
The Wintergreen Group clade of the tribe Gaultherieae (Ericaceae: subfam. Vaccinioideae) comprises the genera Diplycosia Blume, Gaultheria L., and Tepuia Camp. Phylogenetic analysis has demonstrated that Gaultheria is not monophyletic, with Diplycosia and Tepuia nested within it. On morphological grounds, the recognition of a single genus in the Wintergreen Group to establish monophyly as the basis for the classification is favoured over subdivision into smaller genera. Here, we make the taxonomic changes necessary for recognising Gaultheria as the sole genus constituting the Wintergreen Group. We make 126 new combinations, erect 17 new names, and resurrect four species names in Gaultheria for all species, varieties, and forms heretofore recognised in the literature under Diplycosia or Tepuia. Additionally we make two new combinations in Gaultheria at the sectional level to accommodate the species from Diplycosia and Tepuia, and provide lectotypes for Pernettyopsis King \& Gamble and Gaultheria [unranked] Amphicalyx Endl.
\end{abstract}

Keywords. Diplycosia, Gaultherieae, Tepuia, wintergreen

\section{Introduction}

The tribe Gaultherieae (Ericaceae: subfam. Vaccinioideae) comprises seven genera and c. 292 species distributed in East Asia, the Indian Subcontinent, Southeast Asia, Australasia, and the Americas (Middleton, 1991; Argent, 2002; Stevens et al., 2004; Bush et al., 2010; Lu et al., 2019a). Phylogenetic studies based on DNA sequence data have shown that three of these genera, i.e., Gaultheria L., Diplycosia Blume, and Tepuia Camp, form a strongly supported clade known informally as the Wintergreen Group (Kron et al., 1999; Powell \& Kron, 2001; Bush et al., 2009; Fritsch et al., 2011). These studies also show that Gaultheria is not monophyletic and that Diplycosia and Tepuia are nested within it. 
For the classification of the Gaultherieae to be based on monophyly, the number of genera in the Wintergreen Group requires either expansion or reduction. Expansion would impel the recognition of three more genera in the clade, two of which, i.e., the clades corresponding to Gaultheria ser. Hispidulae Airy Shaw and Gaultheria ser. Gymybotrys (Airy Shaw) D.J.Middleton (see Middleton, 1991 and Fritsch et al., 2011), would be straightforward in their morphological definitions. However, the third clade, i.e., that comprising Gaultheria sect. Amblyandra Airy Shaw through the Australia/ New Zealand clade in Fritsch et al. (2011: Fig. 3), has never been named or otherwise recognised in any classification of the Gaultherieae. Moreover, we can detect no solid morphological basis that could warrant its recognition other than a possible reduction in seed size (Lu et al., 2010), despite its strong statistical support in DNA sequencebased phylogenetic studies (Bush et al., 2009; Fritsch et al., 2011; Lu et al., 2019b). We therefore consider it more appropriate to instead recognise the sole genus Gaultheria as comprising the entirety of the Wintergreen Group clade. The recognition of this one large genus is also in line with the general trend of recognising larger plant genera under the criterion of monophyly based at least in part on molecular phylogenetic data (Humphreys \& Linder, 2009). With this circumscription, Gaultheria has the putative morphological synapomorphy of methyl salicylate produced in damaged tissues, and possibly an accrescent mature fleshy calyx and obliquely pyramidal seeds, although all of these features apparently have been lost or modified in some derived lineages within the clade multiple times (Powell \& Kron, 2001; Bush et al., 2009; Lu et al., 2010; Fritsch et al., 2011).

We are preparing a formal revised sectional classification of Gaultheria based on a synthesis of morphological and molecular data. During the process of completing the revision, the need for the timely publication of the revised species taxonomy based on the phylogenetic data has become apparent. For example, ongoing floristic projects such as the Flora of Peninsular Malaysia series (Kiew et al., 2007) often wish to base their taxonomy on monophyletic groups whenever possible. Accordingly, here we provide the new combinations and new and resurrected names in Gaultheria (Ericaceae) resulting from the placement of Diplycosia and Tepuia into the synonymy of Gaultheria.

\section{Methods}

The infrageneric names transferred (species, varieties, and forms) are those of taxa currently recognised in major publications that cover the entirety of the infrageneric taxonomy of Diplycosia and Tepuia. The names transferred from Diplycosia are those accepted in Sleumer's (1957) revision of Diplycosia, Sleumer's Flora Malesiana treatment (1966-1967), and new taxa from the region published since (Argent, 1982, 1989, 2002, 2013, 2014, 2018; Ferreras \& Argent, 2011; Argent \& Widjaja, 2015; Fritsch \& Bush, 2016; Fritsch \& Amoroso, 2016, 2017; Wilkie \& Argent, 2016; Mustaqim \& Ardi, 2019; Mustaqim et al., 2019, 2020). Watthana (2010) and (2015) were also consulted for a more recent treatment of Diplycosia for Thailand. Panda 
et al. (2012) consider D. indica Debta \& H.J.Chowdhery, J. Bot. Res. Inst. Texas 3: 147 (2009) to be a heterotypic synonym of Gaultheria akaensis S.Panda \& Sanjappa, Edinburgh J. Bot. 63: 15 (2006), and so a new combination is not made in Gaultheria for this species. The names transferred from Tepuia are those recognised by Luteyn (1995) in the treatment of the genus for Flora Neotropica. Most of the transferred names retain the original epithet as described in Diplycosia or Tepuia. In cases where a new (replacement) name was required, the epithets often refer to either the locality of the type of the taxon as indicated in the original description, or a botanist in honour of significant contributions to science.

\section{Taxonomy}

Gaultheria L., Sp. P1. 1: 395 (1753). - TYPE: Gaultheria procumbens L.

Brossaea L., Sp. P1. 2: 1190 (1753). - TYPE: Brossaea coccinea L. (= Gaultheria domingensis Urb.).

Hippomanica Molina, Sag. Stor. Nat. Chili 126 (1782). - TYPE: Hippomanica insana Molina (= Gaultheria insana (Molina) D.J.Middleton).

Chiogenes Salisb., Trans. Hort. Soc. London 2: 94 (1817). - TYPE: Vaccinium hispidulum L. (= Gaultheria hispidula (L.) Muhl. ex Bigelow).

Shallonium Raf., Amer. Monthly Mag. \& Crit. Rev. 2(4): 266 (1818). - TYPE: Shallonium serrulatum Raf. (= Gaultheria shallon Pursh).

Pernettya Gaudich., Ann. Sci. Nat. (Paris) 5: 102 (1825) [as 'Pernettia']. - TYPE: Pernettya pumila (L.f.) Hook. (= Gaultheria pumila (L.f.) D.J.Middleton).

Diplycosia Blume, Bijdr. Fl. Ned. Ind. 15: 857 (1826). - TYPE: Diplycosia heterophylla Blume, lectotype designated by Sleumer (1957) (= Gaultheria heterophylla (Blume) Endl. ex Hassk.).

Pernettyopsis King \& Gamble, J. Asiat. Soc. Bengal, Pt. 2, 74: 79 (1906 ['1905']). - TYPE: Pernettyopsis malayana King \& Gamble, lectotype designated here (= Gaultheria macrocalyx Kron \& P.W.Fritsch).

Tepuia Camp in Gleason \& Killip, Brittonia 3: 178 (1939). - TYPE: Tepuia tatei Camp (= Gaultheria campii Kron \& P.W.Fritsch).

Distribution. Approximately 292 species in East Asia (including Japan), Indian Subcontinent, Southeast Asia, Australasia, Americas. 
Gaultheria sect. Diplycosia (Blume) Kron \& P.W.Fritsch, comb. \& stat. nov. Diplycosia Blume, Bijdr. Fl. Ned. Ind. 15: 857 (1826).-TYPE: Diplycosia heterophylla Blume, lectotype designated by Sleumer (1957) (= Gaultheria heterophylla (Blume) Endl. ex Hassk.).

Gaultheria [unranked] Amphicalyx Endl., Gen. P1. 756 (1839). - TYPE: Diplycosia heterophylla Blume, lectotype designated here (= Gaultheria heterophylla (Blume) Endl. ex Hassk.).

Pernettyopsis King \& Gamble, J. Asiat. Soc. Bengal, Pt. 2, 74: 79 (1906 ['1905']). - TYPE: Pernettyopsis malayana King \& Gamble, lectotype designated above (= Gaultheria macrocalyx Kron \& P.W.Fritsch).

Distribution. A section of 125 species in Thailand, Vietnam, Malaysia, Brunei, Indonesia and Papua New Guinea.

Notes. Almost all species previously described in Diplycosia and Pernettyopsis are now accommodated in Gaultheria sect. Diplycosia. As there is no competing name at the rank of section, we make the new combination Gaultheria sect. Diplycosia to preserve the connection to the genus name. "Gaultheria sect. Amphicalyx Endl." was placed in synonymy of Diplycosia by Sleumer (1966-1967) but the name has never been validly published. This name was based on Gaultheria [unranked] Amphicalyx Endl. but as Sleumer included it only in synonymy it was not validated (see Turland et al., 2018: Art. 33.1).

1. Gaultheria abanii (Argent) Kron \& P.W.Fritsch, comb. nov. - Diplycosia abanii Argent, Gard. Bull. Singapore 54: 218 (2002).

2. Gaultheria abscondita (Sleumer) Kron \& P.W.Fritsch, comb. nov. - Diplycosia abscondita Sleumer, Blumea 11: 4 (1961).

3. Gaultheria amboinensis (Becc.) Kron \& P.W.Fritsch, comb. nov. - Diplycosia amboinensis Becc., Malesia 1: 210 (1878).

4. Gaultheria annamensis (Sleumer) Kron \& P.W.Fritsch, comb. nov. - Vaccinium pilosum A.Chev. ex Dop in Lecomte, F1. Indo-Chine 3: 705 (1930), non Gaultheria pilosa (Blume) Zoll. \& Moritzi, Natuur- Geneesk. Arch. Ned.-Indië 2: 9 (1845). Diplycosia annamensis Sleumer, Reinwardtia 4: 139 (1957).

Notes. Sleumer (1957) conferred the name Diplycosia annamensis to this taxon because of the name Diplycosia pilosa Blume, Bijdr. Fl. Ned. Ind. 15: 858 (1826). The epithet 'pilosa' cannot be used in Gaultheria for this species because of Gaultheria pilosa (Blume) Zoll. \& Moritzi (1845). 
5. Gaultheria aperta (J.J.Sm.) Kron \& P.W.Fritsch, comb. nov. - Diplycosia aperta J.J.Sm., Bot. Jahrb. Syst. 68: 208 (1937).

6. Gaultheria apiculifera (J.J.Sm.) Kron \& P.W.Fritsch, comb. nov. - Diplycosia apiculifera J.J.Sm., Repert. Spec. Nov. Regni Veg. 35: 296 (1934).

7. Gaultheria apoensis (Elmer) Kron \& P.W.Fritsch, comb. nov. - Diplycosia apoensis Elmer, Leafl. Philipp. Bot. 3: 1101 (1911).

8. Gaultheria argentii Kron \& P.W.Fritsch, nom. nov. - Diplycosia hirtiflora Argent, Notes Roy. Bot. Gard. Edinburgh 46: 17 (1989), non Gaultheria hirtiflora Benth., P1. Hartw. 66 (1840).

Notes. The new epithet honours the British botanist Dr Graham Charles George Argent (1941-2019), leading authority on the taxonomy of the Ericaceae and who originally named this species.

9. Gaultheria aurea (Sleumer) Kron \& P.W.Fritsch, comb. nov. - Diplycosia aurea Sleumer, Bot. Jahrb. Syst. 71: 148 (1940).

10. Gaultheria balgooyi (Argent) Kron \& P.W.Fritsch, comb. nov. - Diplycosia balgooyi Argent, Edinburgh J. Bot. 71: 88 (2014).

11. Gaultheria barbigera (Sleumer) Kron \& P.W.Fritsch, comb. nov. - Diplycosia barbigera Sleumer, Bot. Jahrb. Syst. 71: 149 (1940).

12. Gaultheria bartolomei (Ferreras \& Argent) Kron \& P.W.Fritsch, comb. nov. Diplycosia bartolomei Ferreras \& Argent, Edinburgh J. Bot. 68: 39 (2011).

13. Gaultheria beamanii Kron \& P.W.Fritsch, nom. nov. - Diplycosia crenulata Sleumer, Bot. Jahrb. Syst. 71: 153 (1940), non Gaultheria crenulata Kurz, J. Bot. 11: 195 (1873).

Notes. The new epithet honours Dr John H. Beaman (1929-2015) for significant contributions to our knowledge of the flora of Mt Kinabalu, to which this species is endemic, and who encouraged many undergraduate and graduate students to pursue plant systematics as a profession.

14. Gaultheria beccarii Kron \& P.W.Fritsch, nom. nov. - Diplycosia acuminata Becc., Malesia 1: 212 (1878), non Gaultheria acuminata Cham. \& Schltdl., Linnaea 5: 126 (1830).

Notes. The new epithet honours the Italian botanist Odoardo Beccari (1843-1920), a contemporary of Charles Darwin and Joseph Hooker, who made significant 
contributions to the botany of Malesia, and who originally named this species and collected its type.

15. Gaultheria benitotanii (Argent) Kron \& P.W.Fritsch, comb. nov. - Diplycosia benitotanii Argent, Philipp. J. Syst. Biol. 12: 73 (2018).

16. Gaultheria benomensis Kron \& P.W.Fritsch, nom. nov. - Diplycosia lancifolia Ridl., J. Straits Branch Roy. Asiat. Soc. 39: 15 (1903), non Gaultheria lancifolia Small, N. Amer. Fl. 29: 78 (1914).

Notes. The new epithet refers to Gunung [Mount] Benom [= Benum], the locality from which the type specimen was collected.

17. Gaultheria benomensis Kron \& P.W.Fritsch var. calvescens (Sleumer) Kron \& P.W.Fritsch, comb. nov. - Diplycosia lancifolia Ridl. var. calvescens Sleumer, Reinwardtia 4: 149 (1957).

18. Gaultheria brachyantha (Sleumer) Kron \& P.W.Fritsch, comb. nov. - Diplycosia brachyantha Sleumer, Reinwardtia 4: 138 (1957).

19. Gaultheria brachyantha (Sleumer) Kron \& P.W.Fritsch var. parvula (Sleumer) Kron \& P.W.Fritsch, comb. nov. - Diplycosia brachyantha Sleumer var. parvula Sleumer, Reinwardtia 4: 139 (1957).

20. Gaultheria capitata (Sleumer) Kron \& P.W.Fritsch, comb. nov. - Diplycosia capitata Sleumer, Bot. Jahrb. Syst. 71: 149 (1940).

21. Gaultheria capitata (Sleumer) Kron \& P.W.Fritsch var. crassiramea (Sleumer) Kron \& P.W.Fritsch, comb. nov. - Diplycosia crassiramea Sleumer, Reinwardtia 4: 141 (1957). - Diplycosia capitata Sleumer var. crassiramea (Sleumer) Argent, Edinburgh J. Bot. 71: 91 (2014).

22. Gaultheria carrii (Sleumer) Kron \& P.W.Fritsch, comb. nov. - Diplycosia carrii Sleumer, Reinwardtia 4: 129 (1957).

23. Gaultheria caryophylloides (J.J.Sm.) Kron \& P.W.Fritsch, comb. nov. - Diplycosia caryophylloides J.J.Sm., Bot. Jahrb. Syst. 68: 209 (1937).

24. Gaultheria caryophylloides (J.J.Sm.) Kron \& P.W.Fritsch var. longipes (Sleumer) Kron \& P.W.Fritsch, comb. nov. - Diplycosia caryophylloides J.J.Sm. var. longipes Sleumer, Reinwardtia 4: 144 (1957).

25. Gaultheria caudatifolia (Sleumer) Kron \& P.W.Fritsch, comb. nov. - Diplycosia caudatifolia Sleumer, Bot. Jahrb. Syst. 71: 150 (1940). 
26. Gaultheria celebensis (J.J.Sm.) Kron \& P.W.Fritsch, comb. nov. - Diplycosia celebensis J.J.Sm., Bull. Jard. Bot. Buitenzorg ser. 3, 1: 406 (1920).

27. Gaultheria chrysothrix (Stapf) Kron \& P.W.Fritsch, comb. nov. - Diplycosia chrysothrix Stapf, Trans. Linn. Soc. London, Bot. 4: 191 (1894).

28. Gaultheria ciliolata (Hook.f.) F.Muell., Trans. Roy. Soc. Victoria 1(2): 21. (1889). - Diplycosia ciliolata Hook.f., Icon. P1. 9: t. 894 (1852).

Notes. Although a combination in Gaultheria already exists for this species, it was recognised in Diplycosia by Sleumer (1957, 1966-1967); it is here returned to Gaultheria.

29. Gaultheria cinnabarina (Sleumer) Kron \& P.W.Fritsch, comb. nov. - Diplycosia cinnabarina Sleumer, Reinwardtia 4: 146 (1957).

30. Gaultheria cinnamomifolia (Stapf) Kron \& P.W.Fritsch, comb. nov. - Diplycosia cinnamomifolia Stapf, Trans. Linn. Soc. London, Bot. 4: 195 (1894).

31. Gaultheria clementium (Sleumer) Kron \& P.W.Fritsch, comb. nov. - Diplycosia clementium Sleumer, Bot. Jahrb. Syst. 71: 151 (1940).

32. Gaultheria coi (Argent) Kron \& P.W.Fritsch, comb. nov. - Diplycosia coi Argent, Philipp. J. Sci. 142 (special issue): 52 (2013) [as D. 'coii'].

33. Gaultheria commutata (Sleumer) Kron \& P.W.Fritsch, comb. nov. - Diplycosia commutata Sleumer, Reinwardtia 4: 153 (1957).

34. Gaultheria consobrina (Becc.) Kron \& P.W.Fritsch, comb. nov. - Diplycosia consobrina Becc., Malesia 1: 211 (1878).

35. Gaultheria edulis (Schltr.) Kron \& P.W.Fritsch, comb. nov. - Diplycosia edulis Schltr., Bot. Jahrb. Syst. 55: 163 (1918).

36. Gaultheria ensifolia (Merr.) Kron \& P.W.Fritsch, comb. nov. - Diplycosia ensifolia Merr., J. Straits Branch Roy. Asiat. Soc. 76: 107 (1917).

37. Gaultheria epiphytica (H.R.Fletcher) Kron \& P.W.Fritsch, comb. nov.-Diplycosia epiphytica H.R.Fletcher, Bull. Misc. Inform. Kew 1936: 40 (1936).

38. Gaultheria eymae Kron \& P.W.Fritsch, nom. nov. - Diplycosia stenophylla Sleumer, Reinwardtia 4: 147 (1957), non Gaultheria stenophylla P.W.Fritsch \& Lu Lu, Phytotaxa 201(1): 22 (2015). 
Notes. The new epithet honours the Dutch botanist Dr Pierre Joseph Eyma (19031945), who collected the type of this species.

39. Gaultheria filipes (Sleumer) Kron \& P.W.Fritsch, comb. nov. - Diplycosia filipes Sleumer, Reinwardtia 4: 133 (1957).

40. Gaultheria fimbriata (Sleumer) Kron \& P.W.Fritsch, comb. nov. - Diplycosia fimbriata Sleumer, Blumea 12: 120 (1963).

41. Gaultheria gajoensis Kron \& P.W.Fritsch, nom. nov. - Diplycosia atjehensis Sleumer, Reinwardtia 4: 132 (1957), non Gaultheria atjehensis J.J.Sm., Repert. Spec. Nov. Regni Veg. 35: 293 (1934).

Notes. The new epithet refers to the Gajo [Gayo] land, the locality of the type collection in Aceh Province on the island of Sumatra, Indonesia.

42. Gaultheria gallowayana (Argent) Kron \& P.W.Fritsch, comb. nov. - Diplycosia gallowayana Argent, Edinburgh J. Bot. 71: 96 (2014).

43. Gaultheria glaucicaulis (Argent) Kron \& P.W.Fritsch, comb. nov. - Diplycosia glaucicaulis Argent, Edinburgh J. Bot. 71: 99 (2014).

44. Gaultheria glauciflora (Sleumer) Kron \& P.W.Fritsch, comb. nov. - Diplycosia glauciflora Sleumer, Reinwardtia 4: 137 (1957).

45. Gaultheria gracilipes (J.J.Sm.) Kron \& P.W.Fritsch, comb. nov. - Diplycosia gracilipes J.J.Sm., Bull. Jard. Bot. Buitenzorg ser. 3, 1: 407 (1920).

46. Gaultheria haemantha (Sleumer) Kron \& P.W.Fritsch, comb. nov. - Diplycosia haemantha Sleumer, Reinwardtia 4: 150 (1957).

47. Gaultheria hendrianiana (Argent) Kron \& P.W.Fritsch, comb. nov. - Diplycosia hendrianiana Argent, Edinburgh J. Bot. 71: 103 (2014) [as D. 'hendriana'].

48. Gaultheria heterophylla (Blume) Endl. ex Hassk., Cat. Hort. Bog., 160 (1844). Diplycosia heterophylla Blume, Bijdr. Fl. Ned. Ind. 15: 858 (1826).

Notes. Although a combination in Gaultheria already exists for this species, it was recognised in Diplycosia by Sleumer (1957, 1966-1967); it is here returned to Gaultheria.

49. Gaultheria heterophylla (Blume) Endl. ex Hassk. var. latifolia (Blume) Kron \& P.W.Fritsch, comb. nov. - Diplycosia latifolia Blume, Bijdr. Fl. Ned. Ind. 15: 858 (1826). - Gaultheria latifolia (Blume) Endl. ex Hassk., Cat. Hort. Bog.: 
160 (1844). - Diplycosia heterophylla Blume var. latifolia (Blume) Sleumer, Reinwardtia 4: 156 (1957).

50. Gaultheria jiewhoei (Mustaqim) Kron \& P.W.Fritsch, comb. nov. - Diplycosia jiewhoei Mustaqim, Telopea 22: 194 (2019).

51. Gaultheria kalimantanensis (P.Wilkie \& Argent) Kron \& P.W.Fritsch, comb. nov. - Diplycosia kalimantanensis P.Wilkie \& Argent, Edinburgh J. Bot. 73: 140 (2016).

52. Gaultheria kalmiifolia (Sleumer) Kron \& P.W.Fritsch, comb. nov. - Diplycosia kalmiifolia Sleumer, Bot. Jahrb. Syst. 71: 154 (1940).

53. Gaultheria kemulensis (J.J.Sm.) Kron \& P.W.Fritsch, comb. nov. - Diplycosia kemulensis J.J.Sm., Bull. Jard. Bot. Buitenzorg ser. 3, 13: 459 (1935).

54. Gaultheria kinabaluensis (Stapf) Kron \& P.W.Fritsch, comb. nov. - Diplycosia kinabaluensis Stapf, Trans. Linn. Soc. London, Bot. 4: 193 (1894).

55. Gaultheria kitangladensis (P.W.Fritsch) Kron \& P.W.Fritsch, comb. nov. Diplycosia kitangladensis P.W.Fritsch, Nat. Hist. Bull. Siam Soc. 62: 22 (2017).

56. Gaultheria kjellbergii (J.J.Sm.) Kron \& P.W.Fritsch, comb. nov. - Diplycosia kjellbergii J.J.Sm., Bot. Jahrb. Syst. 68: 206 (1937).

57. Gaultheria kosteri (Sleumer) Kron \& P.W.Fritsch, comb. nov. - Diplycosia kosteri Sleumer, Blumea 12: 117 (1963).

58. Gaultheria kostermansii (Sleumer) Kron \& P.W.Fritsch, comb. nov. - Diplycosia kostermansii Sleumer, Blumea 11: 5 (1961).

59. Gaultheria lamii (J.J.Sm.) Kron \& P.W.Fritsch, comb. nov. - Diplycosia lamii J.J.Sm., Nova Guinea 18: 99 (1936).

60. Gaultheria lavandulifolia (Sleumer) Kron \& P.W.Fritsch, comb. nov. - Diplycosia lavandulifolia Sleumer, Blumea 12: 121 (1963).

61. Gaultheria ledermannii (Schltr.) Kron \& P.W.Fritsch, comb. nov. - Diplycosia ledermannii Schltr., Bot. Jahrb. Syst. 55: 165 (1918).

62. Gaultheria lilianae (J.J.Sm.) Kron \& P.W.Fritsch, comb. nov.-Diplycosia lilianae J.J.Sm. in Gibbs, Fl. Arfak Mts.: 170 (1917). 
63. Gaultheria loheri (Merr.) Kron \& P.W.Fritsch, comb. nov. - Diplycosia loheri Merr., Philipp. J. Sci. 27: 44 (1925).

64. Gaultheria lorentzii (Koord.) Kron \& P.W.Fritsch, comb. nov. - Diplycosia lorentzii Koord., Nova Guinea 8: 881 (1912).

65. Gaultheria lotungensis (Argent) Kron \& P.W.Fritsch, comb. nov. - Diplycosia lotungensis Argent, Notes Roy. Bot. Gard. Edinburgh 46: 19 (1989).

66. Gaultheria luzonica A.Gray, Proc. Amer. Acad. Arts 5: 324. (1861). - Diplycosia luzonica (A.Gray) Merr., Philipp. J. Sci., C 2: 293 (1907).

Notes. Although originally described in Gaultheria, this species was recognised in Diplycosia by Sleumer (1957, 1966-1967); it is here returned to Gaultheria.

67. Gaultheria luzonica A.Gray var. calelanensis (Elmer) Kron \& P.W.Fritsch, comb. nov. - Diplycosia calelanensis Elmer, Leafl. Philipp. Bot. 3: 1103 (1911) [as D. 'calelanense']. - Diplycosia luzonica (A.Gray) Merr. var. calelanensis (Elmer) Sleumer, Reinwardtia 4: 144 (1957).

68. Gaultheria luzonica A.Gray var. merrittii (Merr.) Kron \& P.W.Fritsch, comb. nov. - Diplycosia merrittii Merr., Philipp. J. Sci., C 2: 293 (1907). - Diplycosia luzonica (A.Gray) Merr. var. merrittii (Merr.) Sleumer, Reinwardtia 4: 144 (1957).

69. Gaultheria luzonica A.Gray var. pubens (Sleumer) Kron \& P.W.Fritsch, comb. nov. - Diplycosia luzonica (A.Gray) Merr. var. pubens Sleumer, Reinwardtia 4: 143 (1957).

70. Gaultheria lysolepis (Sleumer) Kron \& P.W.Fritsch, comb. nov. - Diplycosia lysolepis Sleumer, Bot. Jahrb. Syst. 72: 209 (1942).

71. Gaultheria macrocalyx Kron \& P.W.Fritsch, nom. nov. - Pernettyopsis malayana King \& Gamble, J. Asiat. Soc. Bengal, Pt. 2, Nat. Hist. 74: 79 (1906 ['1905']), non Gaultheria malayana Airy Shaw, Bull. Misc. Inform. Kew 1940: 304 (1941). - Diplycosia malayana (King \& Gamble) Argent, Gard. Bull. Singapore 54: 236 (2002).

Notes. The new epithet refers to the notably large calyx in the flowers of this species.

72. Gaultheria mantorii (Argent) Kron \& P.W.Fritsch, comb. nov. - Diplycosia mantorii Argent, Gard. Bull. Singapore 54: 224 (2002). 
73. Gaultheria kingii (Merr.) Kron \& P.W.Fritsch, comb. nov. - Diplycosia kingii Merr., Pap. Michigan Acad. Sci. 1933, 19: 182 (1934).

Diplycosia microphylla Becc., Malesia 1: 212 (1878).

Diplycosia elliptica Ridl., J. Fed. Malay States Mus. 10: 145 (1920).

Diplycosia cordifolia Ridl., J. Fed. Malay States Mus. 10: 145 (1920).

Notes. Sleumer (1966-1967) treated Diplycosia elliptica Ridl. as a distinct species but Watthana (2015) treated it as a synonym of D. microphylla Becc., considering the differences to be taxonomically trivial. Of the names here considered to be heterotypic synonyms, the specific epithets 'microphylla', 'elliptica' and 'cordifolia' are not available for use in Gaultheria because of the names Gaultheria microphylla Hook.f., Bot. Antarct. Voy. 1: 327 (1846), Gaultheria elliptica Cham., Linnaea 8: 502 (1833), and Gaultheria cordifolia Kunth in Humboldt, Bonpland, \& Kunth, Nov. Gen. Sp. 3: 285 (1819), respectively.

Watthana (2015) excluded Diplycosia cordifolia Ridl. from D. microphylla but did not further comment on its taxonomic status other than to mention that the type may comprise more than one element. We, however, follow Sleumer's (1966-1967) synonymy in treating it is as a synonym of Gaultheria kingii pending further clarification of its status.

74. Gaultheria megabracteata (Argent) Kron \& P.W.Fritsch, comb. nov. Pernettyopsis megabracteata Argent, Bot. J. Linn. Soc. 85: 10 (1982). - Diplycosia megabracteata (Argent) Argent, Gard. Bull. Singapore 54: 237 (2002).

75. Gaultheria mekonggaensis (Argent \& Widjaja) Kron \& P.W.Fritsch, comb. nov. - Diplycosia mekonggaensis Argent \& Widjaja, Edinburgh J. Bot. 72: 239 (2015).

76. Gaultheria memecyloides (Stapf) Kron \& P.W.Fritsch, comb. nov. - Diplycosia memecyloides Stapf, Trans. Linn. Soc. London, Bot. 4: 194 (1894).

77. Gaultheria merrillii Kron \& P.W.Fritsch, nom. nov. - Diplycosia parvifolia Merr., Philipp. J. Sci., C 5: 211 (1910), non Gaultheria parvifolia Small, N. Amer. Fl. 29: 78 (1914).

Notes. The new epithet honours the American Elmer Drew Merrill (1876-1956), renowned botanist and taxonomic expert on the plants of East and Southeast Asia, and who originally named this species and collected its type.

78. Gaultheria microsalicifolia (Argent) Kron \& P.W.Fritsch, comb.nov. - Diplycosia microsalicifolia Argent, Bot. J. Linn. Soc. 85: 8 (1982).

79. Gaultheria minutiflora (Sleumer) Kron \& P.W.Fritsch, comb. nov. - Diplycosia minutiflora Sleumer, Reinwardtia 4: 151 (1957). 
80. Gaultheria minutiflora (Sleumer) Kron \& P.W.Fritsch var. glandulifera (Argent) Kron \& P.W.Fritsch, comb. nov. - Diplycosia minutiflora Sleumer var. glandulifera Argent, Edinburgh J. Bot. 71: 106 (2014).

81. Gaultheria mogeana (Argent) Kron \& P.W.Fritsch, comb. nov. - Diplycosia mogeana Argent, Gard. Bull. Singapore 54: 221 (2002).

82. Gaultheria morobeensis (Sleumer) Kron \& P.W.Fritsch, comb. nov. - Diplycosia morobeensis Sleumer, Bot. Jahrb. Syst. 72: 210 (1942).

83. Gaultheria morobeensis (Sleumer) Kron \& P.W.Fritsch var. ovatifolia (Sleumer) Kron \& P.W.Fritsch, comb. nov. - Diplycosia morobeensis Sleumer var. ovatifolia Sleumer, Reinwardtia 4: 140 (1957).

84. Gaultheria muluensis Kron \& P.W.Fritsch, nom. nov. - Diplycosia salicifolia Sleumer, Blumea 12: 122 (1963), non Gaultheria salicifolia Phil., Anales Univ. Chile 90: 192 (1895).

Notes. The new epithet refers to Mt Mulu, the locality where the type specimen was collected in Sarawak State, Malaysia, on the island of Borneo.

85. Gaultheria muluensis Kron \& P.W.Fritsch f. gigantea (Argent) Kron \& P.W.Fritsch, comb. nov. - Diplycosia salicifolia Sleumer f. gigantea Argent, Gard. Bull. Singapore 54: 227 (2002).

86. Gaultheria muscicola (Sleumer) Kron \& P.W.Fritsch, comb. nov. - Diplycosia muscicola Sleumer, Blumea 12: 118 (1963).

87. Gaultheria myrtillus (Stapf) Kron \& P.W.Fritsch, comb. nov. - Diplycosia myrtillus Stapf, Trans. Linn. Soc. London, Bot. 4: 192 (1894).

88. Gaultheria ngii (Argent) Kron \& P.W.Fritsch, comb. nov. - Diplycosia ngii Argent, Gard. Bull. Singapore 54: 233 (2002).

89. Gaultheria orophila (Sleumer) Kron \& P.W.Fritsch, comb. nov. - Diplycosia orophila Sleumer, Bot. Jahrb. Syst. 71: 155 (1940).

90. Gaultheria othmanii (Argent) Kron \& P.W.Fritsch, comb. nov. - Diplycosia othmanii Argent, Gard. Bull. Singapore 54: 227 (2002).

91. Gaultheria papuana (Mustaqim, Utteridge \& Heatubun) Kron \& P.W.Fritsch, comb. nov. - Diplycosia papuana Mustaqim, Utteridge \& Heatubun, Kew Bull. 74-68: 1 (2019). 
92. Gaultheria paulsmithii (Argent) Kron \& P.W.Fritsch, comb. nov. - Diplycosia paulsmithii Argent, Gard. Bull. Singapore 54: 230 (2002).

93. Gaultheria pendens (Sleumer) Kron \& P.W.Fritsch, comb. nov. - Diplycosia pendens Sleumer, Blumea 12: 119 (1963).

94. Gaultheria penduliflora (Stapf) Kron \& P.W.Fritsch, comb. nov. - Diplycosia penduliflora Stapf, Trans. Linn. Soc. London, Bot. 4: 193 (1894).

95. Gaultheria piceifolia (Sleumer) Kron \& P.W.Fritsch, comb. nov. - Diplycosia piceifolia Sleumer, Reinwardtia 4: 147 (1957).

96. Gaultheria pilosa (Blume) Zoll. \& Moritzi, Natuur-Geneesk. Arch. Ned.-Indië 2: 9 (1845). - Diplycosia pilosa Blume, Bijdr. Fl. Ned. Ind. 15: 858 (1826).

Notes. Although a combination in Gaultheria already exists for this species, it was recognised in Diplycosia by Sleumer (1957, 1966-1967); it is here returned to Gaultheria.

97. Gaultheria pinifolia (Stapf) Kron \& P.W.Fritsch, comb. nov. - Diplycosia pinifolia Stapf, Trans. Linn. Soc. London, Bot. 4: 192 (1894).

98. Gaultheria pittosporifolia (J.J.Sm.) Kron \& P.W.Fritsch, comb. nov. - Diplycosia pittosporifolia J.J.Sm., Bull. Jard. Bot. Buitenzorg ser. 3, 13: 460 (1935).

99. Gaultheria pittosporifolia (J.J.Sm.) Kron \& P.W.Fritsch var. punctiloba (Sleumer) Kron \& P.W.Fritsch, comb. nov. - Diplycosia pittosporifolia J.J.Sm. var. punctiloba Sleumer, Reinwardtia 4: 152 (1957).

100. Gaultheria platyphylla (P.W.Fritsch) Kron \& P.W.Fritsch, comb. nov. - Diplycosia platyphylla P.W.Fritsch, PhytoKeys 69: 33 (2016).

101. Gaultheria puradyatmikai (Mustaqim, Utteridge \& Heatubun) Kron \& P.W.Fritsch, comb. nov. - Diplycosia puradyatmikai Mustaqim, Utteridge \& Heatubun, Phytotaxa 442(2): 53 (2020).

102. Gaultheria pseudorufescens (Sleumer) Kron \& P.W.Fritsch, comb. nov. Diplycosia pseudorufescens Sleumer, Bot. Jahrb. Syst. 71: 157 (1940).

103. Gaultheria pseudorufescens (Sleumer) Kron \& P.W.Fritsch var. elliptifolia (Sleumer) Kron \& P.W.Fritsch, comb. nov.-Diplycosia pseudorufescens Sleumer var. elliptifolia Sleumer, Reinwardtia 4: 136 (1957). 
104. Gaultheria pubivertex (Sleumer) Kron \& P.W.Fritsch, comb. nov. - Diplycosia pubivertex Sleumer, Reinwardtia 4: 131 (1957).

105. Gaultheria punctulata (Stapf) Kron \& P.W.Fritsch, comb. nov. - Diplycosia punctulata Stapf, Trans. Linn. Soc. London, Bot. 4: 193 (1894).

106. Gaultheria retusa (Sleumer) Kron \& P.W.Fritsch, comb. nov. - Diplycosia retusa Sleumer, Bot. Jahrb. Syst. 71: 156 (1940).

107. Gaultheria rhombica (Argent) Kron \& P.W.Fritsch, comb. nov. - Diplycosia rhombica Argent, Gard. Bull. Singapore 54: 223 (2002).

108. Gaultheria rigidifolia (P.W.Fritsch \& C.M.Bush) Kron \& P.W.Fritsch, comb. nov. - Diplycosia rigidifolia P.W.Fritsch \& C.M.Bush, Nordic J. Bot. 34: 699 (2016).

109. Gaultheria rosea (Sleumer) Kron \& P.W.Fritsch, comb. nov. - Diplycosia rosea Sleumer, Reinwardtia 4: 135 (1957).

110. Gaultheria rosmarinifolia (Sleumer) Kron \& P.W.Fritsch, comb. nov. - Diplycosia rosmarinifolia Sleumer, Bot. Jahrb. Syst. 71: 156 (1940).

111. Gaultheria rubella (Sleumer) Kron \& P.W.Fritsch, comb. nov. - Diplycosia rubella Sleumer, Reinwardtia 4: 148 (1957).

112. Gaultheria rubidiflora (J.J.Sm.) Kron \& P.W.Fritsch, comb. nov. - Diplycosia rubidiflora J.J.Sm., Repert. Spec. Nov. Regni Veg. 30: 172 (1932).

113. Gaultheria rufa (Stapf) Kron \& P.W.Fritsch, comb. nov. - Diplycosia rufa Stapf, Trans. Linn. Soc. London, Bot. 4: 191 (1894).

114. Gaultheria rupicola (Sleumer) Kron \& P.W.Fritsch, comb. nov. - Diplycosia rupicola Sleumer, Reinwardtia 4: 140 (1957).

115. Gaultheria sagittanthera (J.J.Sm.) Kron \& P.W.Fritsch, comb. nov. - Diplycosia sagittanthera J.J.Sm., Bot. Jahrb. Syst. 68: 205 (1937).

116. Gaultheria sanguinolenta (Sleumer) Kron \& P.W.Fritsch, comb. nov. - Diplycosia sanguinolenta Sleumer, Bot. Jahrb. Syst. 71: 157 (1940).

117. Gaultheria saurauioides (J.J.Sm.) Kron \& P.W.Fritsch, comb. nov. - Diplycosia saurauioides J.J.Sm., Bull. Jard. Bot. Buitenzorg ser. 3, 13: 456 (1935).

118. Gaultheria scabrida (Becc.) Kron \& P.W.Fritsch, comb. nov. - Diplycosia scabrida Becc., Malesia 1: 211 (1878). 
119. Gaultheria schramii (Sleumer) Kron \& P.W.Fritsch, comb. nov. - Diplycosia schramii Sleumer, Blumea 12: 116 (1963).

120. Gaultheria schultzei (Schltr.) Kron \& P.W.Fritsch, comb. nov. - Diplycosia schultzei Schltr., Bot. Jahrb. Syst. 55: 163 (1918).

121. Gaultheria sepikensis Kron \& P.W.Fritsch, nom. nov. - Diplycosia rufescens Schltr., Bot. Jahrb. Syst. 55: 165 (1918), non Gaultheria rufescens DC., Prodr. 7: 595 (1839).

Notes. The new epithet refers to the area of Papua New Guinea known as the Sepik region, to which the species is endemic.

122. Gaultheria setiloba (Sleumer) Kron \& P.W.Fritsch, comb. nov. - Diplycosia setiloba Sleumer, Blumea 12: 121 (1963).

123. Gaultheria smithii Kron \& P.W.Fritsch, nom. nov. - Diplycosia setosa J.J.Sm., Bull. Jard. Bot. Buitenzorg ser. 2, 8: 51 (1912), non Gaultheria setosa Small, N. Amer. F1. 29: 77 (1914).

Notes. The new epithet honours the Dutch botanist Johannes Jacobus Smith (1867-1947), who named many species of Malesian Ericaceae, including this species.

124. Gaultheria soror (Becc.) Kron \& P.W.Fritsch, comb. nov. - Diplycosia soror Becc., Malesia 1: 210 (1878).

125. Gaultheria soror (Becc.) Kron \& P.W.Fritsch var. nuda (Sleumer) Kron \& P.W.Fritsch, comb. nov. - Diplycosia soror Becc. var. nuda Sleumer, Blumea 12: 118 (1963).

126. Gaultheria sphenophylla (Sleumer) Kron \& P.W.Fritsch, comb. nov. - Diplycosia sphenophylla Sleumer, Bot. Jahrb. Syst. 71: 158 (1940).

127. Gaultheria steenisii Kron \& P.W.Fritsch, nom. nov. - Diplycosia tetramera Sleumer, Reinwardtia 4: 136 (1957), non Gaultheria tetramera W.W.Sm., Notes Roy. Bot. Gard. Edinburgh 11: 211 (1920).

Notes. The new epithet honours the Dutch botanist Dr Cornelis Gijsbert Gerrit Jan van Steenis (1901-1986), renowned expert on the flora of Malesia and general editor of the Flora Malesiana series, who collected the type of this species.

128. Gaultheria stellaris (Sleumer) Kron \& P.W.Fritsch, comb. nov. - Diplycosia stellaris Sleumer, Blumea 11: 6 (1961). 
129. Gaultheria subglobularis (Sleumer) Kron \& P.W.Fritsch, comb. nov. - Diplycosia subglobularis Sleumer, Blumea 11: 5 (1961).

130. Gaultheria sulawesiensis Kron \& P.W.Fritsch, nom. nov. - Diplycosia hirsuta Sleumer, Bot. Jahrb. Syst. 71: 154 (1940), non Gaultheria hirsuta M.Martens \& Galeotti, Bull. Acad. Roy. Sci. Bruxelles 9: 540 (1842).

Notes. The new epithet refers to the Indonesian island of Sulawesi, to which the species is endemic.

131. Gaultheria sulcinervia (Sleumer) Kron \& P.W.Fritsch, nom. nov. - Diplycosia viridiflora Sleumer, Bot. Jahrb. Syst. 71: 159 (1940), non Gaultheria viridiflora Sleumer, Reinwardtia 4: 176 (1957).

Notes. The new epithet refers to the deeply sulcate midvein of the leaf blade adaxially in this species.

132. Gaultheria sulcinervia (Sleumer) Kron \& P.W.Fritsch var. megalantha (Sleumer) Kron \& P.W.Fritsch, comb. nov. - Diplycosia viridiflora Sleumer var. megalantha Sleumer, Reinwardtia 4: 155 (1957).

133. Gaultheria sumatrensis (Merr.) Kron \& P.W.Fritsch, comb. nov. - Diplycosia sumatrensis Merr., Pap. Michigan Acad. Sci. 1933, 19: 181 (1934).

134. Gaultheria supyanii (Argent) Kron \& P.W.Fritsch, comb. nov. - Diplycosia supyanii Argent, Edinburgh J. Bot. 71: 111 (2014).

135. Gaultheria triangulanthera (J.J.Sm.) Kron \& P.W.Fritsch, comb. nov. Diplycosia triangulanthera J.J.Sm., Bot. Jahrb. Syst. 68: 207 (1937).

136. Gaultheria trinervia (Elmer) Kron \& P.W.Fritsch, comb. nov. - Diplycosia trinervia Elmer, Leafl. Philipp. Bot. 3: 1102 (1911).

137. Gaultheria undata (J.J.Sm.) Kron \& P.W.Fritsch, comb. nov. - Diplycosia undata J.J.Sm., Repert. Spec. Nov. Regni Veg. 30: 171 (1932).

138. Gaultheria urceolata (Stapf) Kron \& P.W.Fritsch, comb. nov. - Diplycosia urceolata Stapf, Trans. Linn. Soc. London, Bot. 4: 191 (1894).

139. Gaultheria urdanetensis (Elmer) Kron \& P.W.Fritsch, comb. nov. - Diplycosia urdanetensis Elmer, Leafl. Philipp. Bot. 7: 2628 (1915). - Diplycosia trinervia Elmer var. urdanetensis (Elmer) Sleumer, Reinwardtia 4: 143 (1957). 
140. Gaultheria varians (Sleumer) Kron \& P.W.Fritsch, comb. nov. - Diplycosia varians Sleumer, Blumea 12: 342 (1964).

Gaultheria sect. Tepuia (Camp) Kron \& P.W.Fritsch, comb. \& stat. nov. - Tepuia Camp in Gleason \& Killip, Brittonia 3: 178 (1939). - TYPE: Tepuia tatei Camp (= Gaultheria campii Kron \& P.W.Fritsch).

Distribution. A section of seven species from the Guayana Highlands of southeastern Venezuela (Bolívar State).

1. Gaultheria auyantepuiensis Kron \& P.W.Fritsch, nom. nov. - Tepuia intermedia Steyerm., Acta Bot. Venez. 2: 303 (1967), non Gaultheria intermedia J.J.Sm., Meded. Rijks-Herb. 30: 1 (1916).

Notes. The new epithet refers to Auyán-tepui, the table-top mountain to which this species is endemic.

2. Gaultheria campii Kron \& P.W.Fritsch, nom. nov. - Tepuia tatei Camp in Gleason \& Killip, Brittonia 3: 179 (1939), non Gaultheria tatei A.C.Sm., Bull. Torrey Bot. Club 60: 99 (1933).

Notes. The new epithet honours Dr W.H. Camp (1904-1963) for his important contributions to the Modern Synthesis unified theory of evolution and our understanding of Ericaceae systematics, and who originally named this species.

3. Gaultheria cardonae (A.C.Sm.) Kron \& P.W.Fritsch, comb. nov. - Tepuia cardonae A.C.Sm., Contr. U.S. Natl. Herb. 29: 338 (1950).

4. Gaultheria empetrifolia Kron \& P.W.Fritsch, nom. nov. - Tepuia venusta Camp in Gleason \& Killip, Brittonia 3: 181 (1939), non Gaultheria venusta DC., Prodr. 7: 597 (1839).

Notes. The new epithet refers to the leaves of this species, which superficially resemble those of Empetrum nigrum L.

5. Gaultheria multiglandulosa (Steyerm. \& Maguire) Kron \& P.W.Fritsch, comb. nov. - Tepuia multiglandulosa Steyerm. \& Maguire, Acta Bot. Venez. 2: 301 (1967).

6. Gaultheria speciosa (A.C.Sm.) Kron \& P.W.Fritsch, comb. nov. - Tepuia speciosa A.C.Sm., Contr. U.S. Natl. Herb. 29: 336 (1950).

7. Gaultheria vareschii (Steyerm.) Kron \& P.W.Fritsch, comb. nov. - Tepuia vareschii Steyerm., Acta Bot. Venez. 2: 306 (1967). 
ACKNOWLEDGEMENTS. We thank Dr David Middleton for helpful comments on the manuscript. This work was supported by U.S. National Science Foundation grants DEB0103795 and DEB-0717711; National Geographic Society grant 148057; National Natural Science Foundation of China grants 41671052 and 31960080; and Program Innovative Research Team in Science and Technology in Yunnan Province 202005AE160004.

\section{References}

Argent, G.C.G. (1982). New taxa and combinations and comments on Ericaceae. Bot. J. Linn. Soc. $85: 2-17$.

Argent, G.C.G. (1989). Diplycosia in Borneo. Two new species and a provisional key to Diplycosia and close allies. Notes Roy. Bot. Gard. Edinburgh 46: 17-26.

Argent, G.C.G. (2002). New taxa and combinations in the genus Diplycosia (Ericaceae) of Borneo and Peninsular Malaysia. Gard. Bull. Singapore 54: 217-238.

Argent, G.C.G. (2013). A new species of Diplycosia: D. coii Argent (Ericaceae) is described in honour of Leonardo Co. Philipp. J. Sci.142(Special Issue): 51-55.

Argent, G. (2014). A contribution to the study of the genus Diplycosia (Ericaceae) in Sulawesi, Indonesia. Edinburgh J. Bot. 71: 83-115.

Argent, G. (2018). A new species of Diplycosia: D. benitotanii Argent (Ericaceae) from Mt. Halcon in the Philippines is described in honour of the late Dr. Benito Tan. Philipp. J. Syst. Biol. 12: 73-76.

Argent, G. \& Widjaja, E.A. (2015). Diplycosia mekonggaensis (Ericaceae, Gaultherieae), a new species from Sulawesi, Indonesia. Edinburgh J. Bot. 72: 239-242.

Bush, C.M., Lu, L., Fritsch, P.W., Li, D.-Z. \& Kron, K.A. (2009). Phylogeny of Gaultherieae (Ericaceae: Vaccinioideae) based on DNA sequence data from $m a t K, n d h F$, and nrITS. Int. J. Pl. Sci. 170: 355-364.

Bush, C.M., Judd, W.S., Frazier, L. \& Kron, K.A. (2010). The phylogeny of Leucothoë s.l. (Ericaceae: Vaccinioideae) based on morphological and molecular ( $n d h F, m a t K$, and nrITS) data. Syst. Bot. 35: 201-206.

Ferreras, U.F. \& Argent, G.C.G. (2011). Diplycosia bartolomei (Ericaceae), a new species from the Philippines. Edinburgh J. Bot. 68: 39-42.

Fritsch, P.W. \& Amoroso, V.B. (2016). Diplycosia platyphylla (Ericaceae), a new species from Mindanao, Philippines. PhytoKeys 69: 31-38.

Fritsch, P.W. \& Amoroso, V.B. (2017). Diplycosia kitangladensis sp. nov. from Mindanao, the Philippines, and a taxonomic reassessment of D. trinervia. Nat. Hist. Bull. Siam Soc. 62: 21-27.

Fritsch, P.W. \& Bush, C.M. (2016). Diplycosia rigidifolia sp. nov. (Ericaceae) from Borneo, Sabah, Malaysia. Nordic J. Bot. 34: 699-702.

Fritsch, P.W., Lu, L., Bush, C.M., Cruz, B.C., Kron, K.A. \& Li, D.Z. (2011). Phylogenetic analysis of the Wintergreen Group (Ericaceae) based on six genic regions. Syst. Bot. 36: 990-1003.

Humphreys, A.M. \& Linder, H.P. (2009). Concept versus data in delimitation of plant genera. Taxon 58: 1054-1074.

Kiew, R., Chung, R.C.K, Saw, L.G. \& Rafidah, R. (2007). The Flora of Peninsular Malaysia project. In: Conference on Forestry \& Forest Products Research 2007, pp. 16-25. Kepong, Kuala Lumpur: Forest Research Institute Malaysia.

Kron, K.A., Judd, W.S. \& Crayn, D.M. (1999). Phylogenetic analyses of Andromedeae (Ericaceae subfam. Vaccinioideae). Amer. J. Bot. 86: 1290-1300. 
Lu, L., Fritsch, P.W., Bush, C.M., Dong, L.-N., Wang, H. \& Li, D.-Z. (2010). Systematic implications of seed coat diversity in Gaultherieae (Ericaceae). Bot. J. Linn. Soc. 162: 477-495.

Lu, L., Fritsch, P.W., Matzke, N.J., Wang, H., Kron, K.A., Li, D.-Z. \& Wiens, J.J. (2019a). Why is fruit colour so variable? Phylogenetic analyses reveal relationships between fruit-colour evolution, biogeography and diversification. Global Ecol. Biogeogr. 28: 891-903.

Lu, L., Fritsch, P.W., Bush, C.M., Wang, H., Kron, K.A. \& Li, D.-Z. (2019b). Allopolyploidy in the Wintergreen Group of tribe Gaultherieae (Ericaceae) inferred from low-copy nuclear genes. Nordic J. Bot. 2019: e02077.

Luteyn, J.L. (1995). Tepuia. In: J.L. Luteyn (ed.) Flora Neotropica Monograph, Monograph 66. Ericaceae Part II. The Superior-Ovaried Genera, pp. 351-364. New York: New York Botanical Garden.

Middleton, D.J. (1991). Infrageneric classification of the genus Gaultheria L. (Ericaceae). Bot. J. Linn. Soc. 106: 229-258.

Mustaqim, W.A. \& Ardi, W.H. (2019). Ericaceae of Sulawesi: A new species of Diplycosia, a new variety of Vaccinium paludicolum and one rediscovery. Telopea 22: 193-204.

Mustaqim, W.A., Utteridge, T.M.A. \& Heatubun, C.D. (2019). Diplycosia papuana (Ericaceae: Vaccinioideae: Gaultherieae): A new endemic species from central New Guinea. Kew Bull. 74: 68.

Mustaqim, W.A., Chikmawati, T., Utteridge, T.M. \& Heatubun, C.D. (2020). A new species of Diplycosia (Ericaceae) from Mount Jaya, western New Guinea. Phytotaxa 442(2): $52-60$.

Panda, S., Reveal, J.L. \& Sanjappa, M. (2012). Reduction of Diplycosia indica (2009) to Gaultheria akaensis (2006) (Ericaceae). Phytoneuron 2012-35: 1-7.

Powell, E.A. \& Kron, K.A. (2001). An analysis of the phylogenetic relationships in the wintergreen group (Diplycosia, Gaultheria, Pernettya, Tepuia; Ericaceae). Syst. Bot. 26: $808-817$.

Sleumer, H. (1957). Florae Malesianae praecorsores XIV. A revision of the genus Diplycosia (Ericaceae). Reinwardtia 4: 119-161.

Sleumer, H. (1966-1967). Ericaceae. In: van Steenis, C.G.G.J. (ed.) Flora Malesiana, ser. 1, Seed Plants, vol. 6, pp. 469-914. Groningen, Netherlands: Wolters-Noordhoff.

Stevens, P.F., Luteyn, J., Oliver, E.G.H., Bell, T.L., Brown, E.A., Crowden, R.K., George, A.S., Jordan, G.J., Ladd, P., Lemson, K., McLean, C.B., Menadue, Y., Pate, J.S., Stace, H.M. \& Weiller, C.M. (2004). Ericaceae. In: Kubitzki, K. (ed.) The Families and Genera of Vascular Plants, vol. 6, pp. 145-194. Berlin: Springer-Verlag.

Turland, N.J., Wiersema, J.H., Barrie, F.R., Greuter, W., Hawksworth, D.L., Herendeen, P.S., Knapp, S., Kusber, W.-H., Li, D.-Z., Marhold, K. et al. (eds) (2018). International Code of Nomenclature for algae, fungi, and plants (Shenzhen Code) adopted by the Nineteenth International Botanical Congress Shenzhen, China, July 2017. Regnum Vegetabile 159. Glashütten: Koeltz Botanical Books.

Watthana, S. (2010). The genus Diplycosia Blume (Ericaceae) in Thailand. Thai J. Bot. 2: $127-132$.

Watthana, S. (2015). Ericaceae. In: Newman, M. \& Barfod, A. (eds) Flora of Thailand, vol. 13, part 1, pp. 101-141. Bangkok: The Forest Herbarium, Department of National Parks, Wildlife and Plant Conservation.

Wilkie, P. \& Argent, G.C.G. (2016). A new species of Diplycosia (Ericaceae) from South Kalimantan, Indonesia. Edinburgh J. Bot. 73: 139-142. 
